\title{
Design and Characterisation of Micro-Diaphragm for Low Power Drug Delivery Applications
}

\author{
Don W. Dissanayake ${ }^{a, b}$, Said F. Al-Sarawi ${ }^{a, b}$, Tien-Fu Lu ${ }^{c}$ and Derek Abbott ${ }^{a, b, c}$ \\ ${ }^{a}$ Centre for High Performance Integrated Technologies and Systems (CHiPTec), \\ University of Adelaide, SA 5005, Australia \\ ${ }^{b}$ School of Electrical and Electronic Engineering, University of Adelaide, SA 5005, Australia \\ ${ }^{c}$ School of Mechanical Engineering, University of Adelaide, SA 5005, Australia \\ ${ }^{d}$ Centre of Biomedical Engineering (CBME), University of Adelaide, SA 5005, Australia
}

\begin{abstract}
Micro-fabricated diaphragms can be used to provide pumping action in microvalve and microfluidic applications. In this paper, a design for a micro-diaphragm that features low power and small area is presented. The diaphragm is actuated using a Surface Acoustic Wave (SAW) device that is interrogated from an RF signal to provide secure actuation operation. The micropump is targeted for in vivo nano-scale drug delivery and similar applications. For low power micropump operation, it is important to design the diaphragm with a higher flexibility while maintaining the stability. Analysis is carried out using ANSYS simulation tools with different design methods and materials. Results achieved from analytical and Finite Element Modeling (FEM) methods are compared and discussed to decide on optimal dimensions for the diaphragm.
\end{abstract}

Keywords: Diaphragm, SAW device, Microvalve, Micropump, Electrostatic, ANSYS, FEM

\section{INTRODUCTION}

In future Micro Electro Mechanical Systems (MEMS), secure, wireless interrogation will become key design issues apart from low power operation and small device size, especially in devices designed for biomedical and related applications. Interestingly, the nanoliter fluid manipulation is a fundamental function that has a wide range of applications such as drug delivery, fertility control, parallel mixing of photolithographically defined nanoliter volumes, lab-on-a-chip applications, precision manufacturing and miniaturisation of chemical and bio-analysis systems. ${ }^{1,2}$ With miniaturization, physical scaling laws inherently favour some technologies and phenomena over others. In some cases, technologies that can be made by micromachining work well at the microscopic scale, but have no analogy or usefulness in the macroscopic domain. ${ }^{3}$

\subsection{Microfluidic Devices for Drug Delivery}

Design and analysis of a microdiaphragm for microvalve and micropump devices is presented in this paper. Such microfluidic devices are mainly intended to use in drug delivery applications where the device needs to be implanted in the human body with a wireless interrogation system. It is highly beneficial to incorporate the pumping action into the microfluidic device as it reduces the dependency of the operation of the device, on the surrounding fluid flow as well as causing the fluid to propagate from the inlet to the outlet. ${ }^{1}$ Based on the previous work carried out by the authors ${ }^{1,3,4}$ it is been demonstrated that the required wireless and batteryless actuation for a microfluidic device is achievable using a fully passive system.

Initially, the micropump structure is designed by integrating a SAW device and a micropump chamber, which causes the microfluidic modulation. The pumping chamber is a suspended structure above the output Inter Digital Transducers (IDT) ${ }^{1}$ of the SAW device. The chamber consists of two diffusers to allow the fluid flow, a thin diaphragm, and the surrounding walls to form the required enclosure. The diaphragm is considered

Further author information: (Send correspondence to Don W. Dissanayake)

Don W. Dissanayake: E-mail: don.dissanayake@adelaide.edu.au

Active and Passive Smart Structures and Integrated Systems 2008, edited by Mehdi Ahmadian

Proc. of SPIE Vol. 6928, 69282P, (2008) ·0277-786X/08/\$18 - doi: 10.1117/12.790789

Proc. of SPIE Vol. 6928 69282P-1 
to be made out of a flexible conductive material such as silicon. ${ }^{1}$ In order to increase the pumping effect and gain higher flow rates, a novel micropump is designed with two SAW devices and two diaphragms as shown in Figure 1. Such a device is capable of modulating the fluid flow with a better control as more power is absorbed from the interrogating electromagnetic field, through two SAW devices, in contrast to the initial design, which only consist of one SAW device.

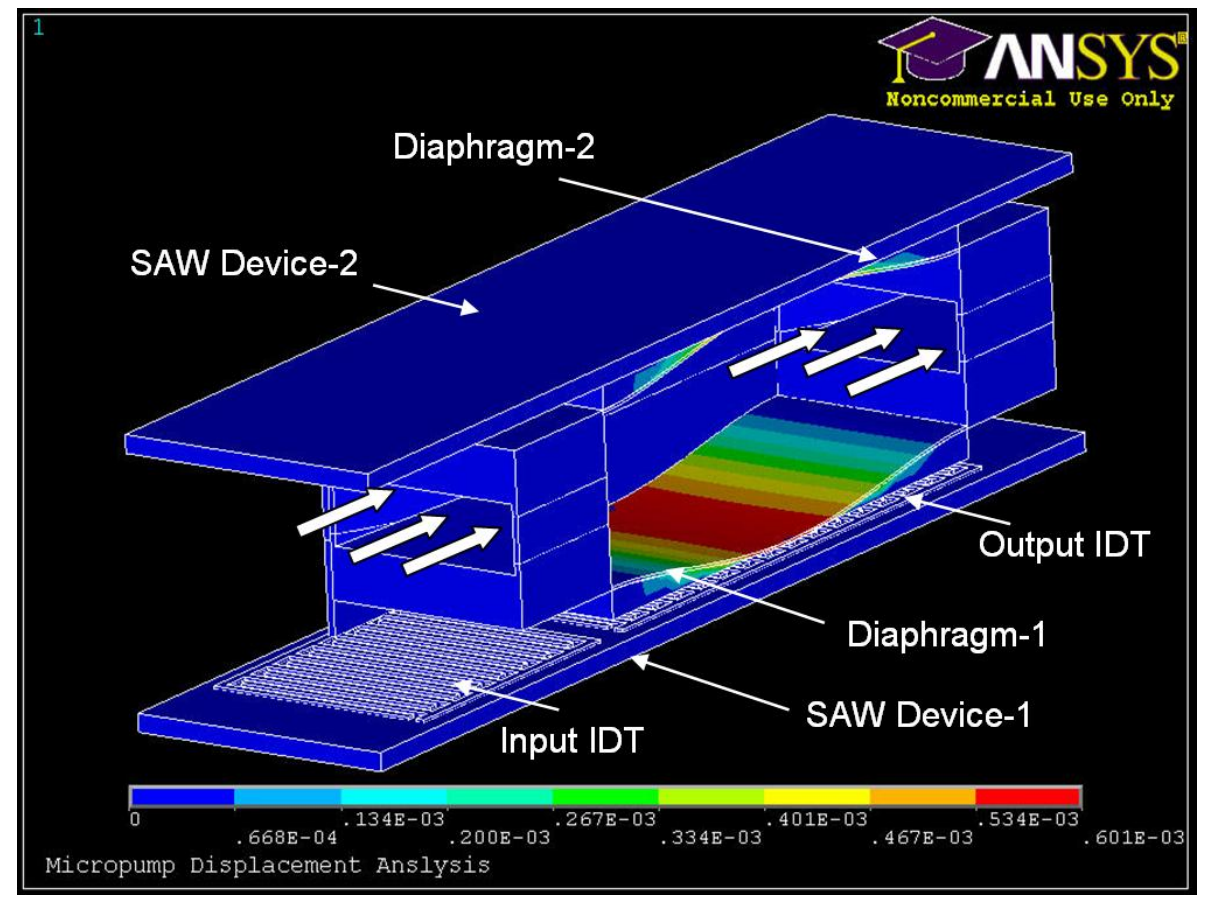

Figure 1. Micropump structure with two SAW devices and two diaphragms, which are used to pump the fluid through at a higher flow rate.

The SAW device based microdiaphragm actuation is discussed in Section 2. In Section 3, critical parameters that need to be considered, when designing diaphragms, are presented and incorporation of corrugations to a diaphragm is also illustrated. Then in Section 4, the important steps associated with FEA are highlighted and the simulation results are presented.

\section{SAW DEVICE BASED DIAPHRAGM ACTUATION}

Surface Acoustic Wave devices are widely used in MEMS related applications, which require secure, wireless, and passive interrogation. ${ }^{5}$ These devices are recognized for their versatility and efficiency in controlling and processing electrical signals. They are based on propagation of acoustic waves in elastic solids and the coupling of these waves to electric charge signals via input and output IDTs deposited on a piezoelectric material. Basically, we may think of a SAW device as consisting of a solid substrate with input and output IDTs. ${ }^{5}$ As depicted in Figure 1, two microdiaphragms are electrostatically actuated using two SAW devices.

The operating principle of the device can be elaborated using one SAW device and a diaphragm. The placement of the conductive diaphragm above the SAW device is shown in Figure 2. The input IDT converts the incoming RF signal by the inverse piezoelectric effect into SAWs, which propagate along the planar surface of the piezoelectric substrate. At the output IDT, the SAWs are reconverted to an electrical signal because of the piezoelectric effect. The conductive diaphragm (an equipotential plate) is suspended a few micrometers above the output IDT. Therefore an electrostatic field is generated between the output IDT and the diaphragm and the resulting electrostatic force deflects the thin diaphragm. 


\section{SIDE VIEW:}

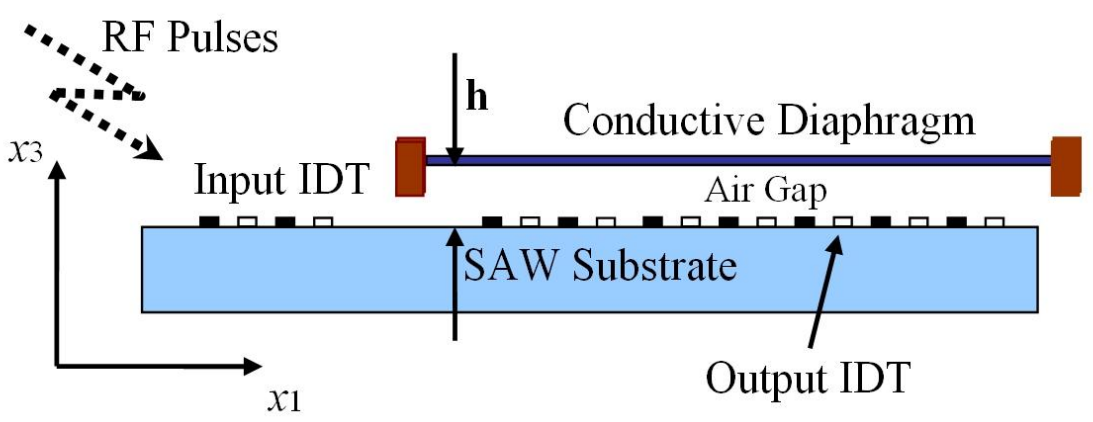

TOP VIEW:

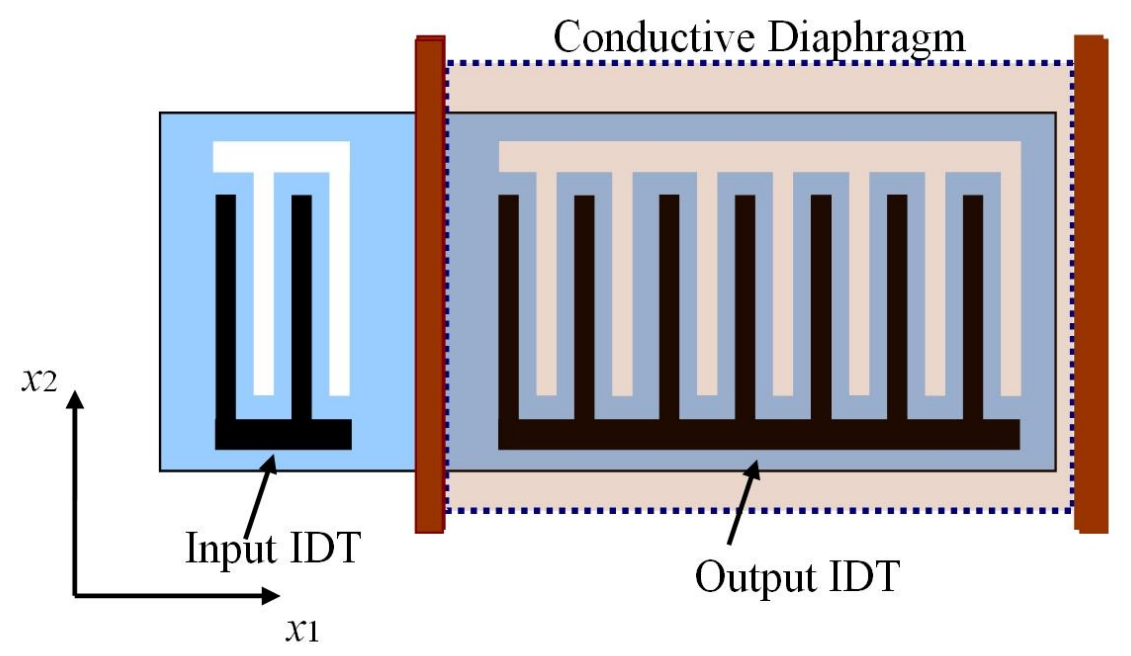

Figure 2. Side view: Air-gap separated diaphragm is placed above the output IDT of the SAW device. Top view: The area of the diaphragm is larger than the effective area of the output IDT, hence allows more deflection as the stress levels at the central area of the diaphragm is less.

\section{DIAPHRAGM DESIGN}

A diaphragm is essentially a thin circular or rectangular plate, made out of a flexible material, stretched and fastened at its periphery. Diaphragms are widely used as sensing elements in MEMS devices, for high accuracy and good dynamic response. Generally the main characteristics of a diaphragm are ruggedness, excellent stability and reliability, low hysteresis and creep, and good dynamic response. Moreover, other characteristics such as effect of the environmental conditions, material of the diaphragm, size, weight, and fabrication technology vary enormously, depending on the transduction * principle in use, range, and actual application. ${ }^{6,7}$

The main considerations while selecting a suitable diaphragm material for drug delivery applications are the chemical nature of the fluid/drug that is expected to come in contact with the diaphragm, temperature range, effects of shock and vibration, and frequency response requirements. ${ }^{6}$ Performance of the diaphragm can be improved when it is cycled a number of times over the expected range of operating temperatures. It is important to incorporate the pressure cycling and heat treatment, at the manufacturing stage, to reduce the elastic-after

\footnotetext{
${ }^{*}$ Transduction - The process whereby a transducer accepts energy in one form and gives back related energy in a different form.
} 
effects of the diaphragm. In this way, the consistency and the reproducibility of the diaphragm operation can be increased. ${ }^{6,7}$ Moreover the mechanical sensitivity of the diaphragm can be improved by both releasing the initial stress and reducing the bending stiffness of the diaphragm. ${ }^{8}$

\subsection{Displacement Enhancement Methods}

For most thin diaphragms, however, large tensile stress can lead to undesirable effects such as low and irreproducible performances, if the processes cannot accurately be controlled. As a result the displacement characteristics can be affected as well. In order to design diaphragms with low-stress and higher displacements, various design techniques can be followed. One such technique is to use a sandwich structure, in which layers with compressive and tensile stress are combined. Another stress releasing technique is to form corrugations in the diaphragm. The corrugated diaphragm has been verified to be capable of releasing the built-in stress, thereby increasing the mechanical sensitivity of the diaphragm and reducing the irreproducibility. ${ }^{8}$

Compared with the conventional flat diaphragm, the shallowly corrugated diaphragms have demonstrated improved sensitivity, especially for a high residual stress level. Although shallow corrugation technique can sufficiently reduce the membrane rigidity of the diaphragm, the bending rigidity, however, may be largely increased due to the increased 'equivalent thickness' of the diaphragm. ${ }^{8}$ The effect of the corrugated profiles in achieving higher displacements is further discussed and analysed in the following subsection.

\subsection{Corrugated Diaphragm Design}

The structure of the diaphragm may be flat or corrugated depending on the intended MEMS application. Flat diaphragms are used for most of the sensing related applications. However for applications where large displacements are desired, corrugated diaphragm structures are ideally suited. ${ }^{6}$ A corrugated diaphragm is made by forming concentric beads or corrugations on a thin sheet of metal (or a similar material). As a result, diaphragm increases in rigidity and becomes suitable for operation at larger displacements and longer linear travel than a flat diaphragm. ${ }^{9}$ As it can be seen from the Figure 3, corrugated diaphragms can take various forms.

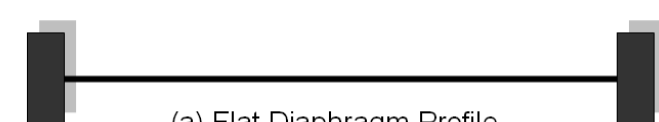

(a) Flat Diaphragm Profile

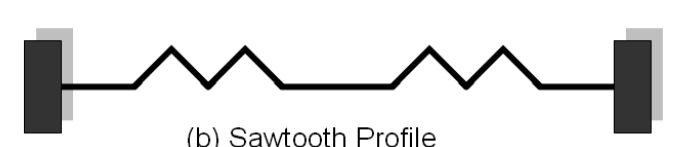

(b) Sawtooth Profile

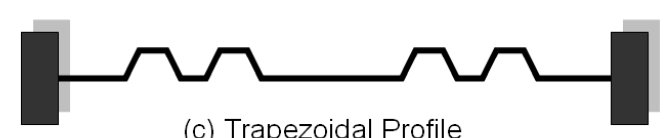

(c) Trapezoidal Profile

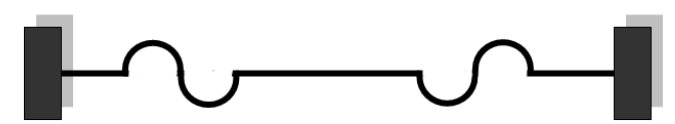

(d) Sinusoidal Profile

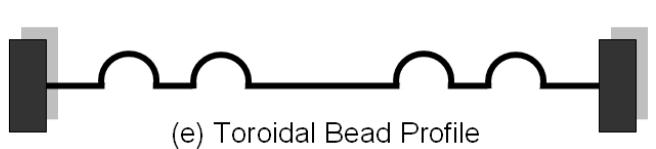

Figure 3. Different types of corrugated profiles.

These diaphragms can be operated either as a single element or for higher sensitivity as a capsule, having two diaphragms facing one another. Similar logic is applicable when diaphragms are used as actuators for micropumps, since higher displacements are achievable from the capsule like designs. It has been suggested 
that the shape of the profile has little effect on the performance characteristics of the diaphragm. ${ }^{9}$ In general, diaphragms with fine sawtooth profiles are simple to manufacture and are stable at small overloads. However, manufacture of diaphragms with deep sawtooth profiles is comparatively difficult due to the possibility of cracks developing at crests due to stress concentration. Whereas, the fabrication of diaphragms having sinusoidal and similar shaped corrugations needs more complex tools. But in such corrugation profiles, the stress concentration is minimal compared to sawtooth and trapezoidal profiles. As a result, a sinusoidal profile is selected and analysed in the proposed diaphragm design.

\subsection{Consideration of Parameters for Sinusoidal Corrugation Design}

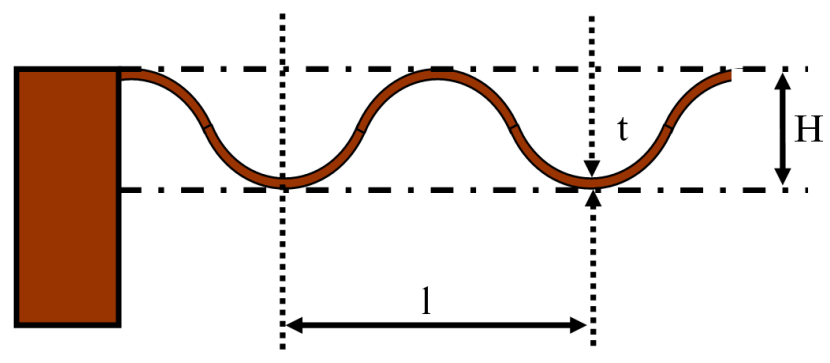

Figure 4. The standard sinusoidal corrugations. Important parameters for sinusoidal profile are $l$ : Wave length of the corrugations, $H$ : Total depth of convolution, and $t$ : Thickness of the diaphragm.

Parameters $l, H$ and $t$ are considered to be the critical parameters in the design of the sinusoidal corrugations as depicted in Figure 4. The ratios $H / t$, and $l / H$ need to be carefully considered to reduce the unnecessary stress concentration. ${ }^{9}$ Moreover in this specific design, more displacement is achieved by reducing $h$, the allowable gap between the diaphragm and the SAW device (see Figure 2), as the electrostatic force is inversely proportionated to the gap between the electrodes- $h$. As it was highlighted in Figure 2, a diaphragm with a larger area results in producing higher displacements around the central region of the diaphragm. However, the dimensions of the diaphragm should be not too large, to avoid overlapping with the input IDT. Considering all these facts, different corrugated diaphragms are designed and analysed using ANSYS simulation tools, ${ }^{10}$ and presented in Section 4.

\section{FEA AND SIMULATION RESULTS}

A sequential procedure can be followed in design and analysis of electrostatically actuated diaphragms in ANSYS tools. ${ }^{1}$ As it was presented in authors' previous work, ${ }^{1,3}$ only the effect of the output IDT is considered for the FEA, instead of incorporating the whole SAW device.

Once the geometry is created using pre-decided micro dimensions, element types and material properties are defined for the diaphragm and the air-gap. Here, SOLID95 and SOLID122 are chosen as the element types for the diaphragm and the air-gap, respectively. This is mainly because these elements can tolerate irregular shapes without as much loss of accuracy, capable of having compatible displacement shapes, and well suited to model curved boundaries. Material properties of silicon are chosen for the diaphragm and properties of air are used for the gap between the electrodes. Next, the created geometry is meshed to a reasonably fine level to accommodate for accurate micro level changes in the structure. Care is taken while deciding on meshing parameters, so that accurate results are obtained in less simulation time. Once the geometry is meshed, electric and mechanical boundary conditions are applied by picking either a set of relevant nodes or elements.

Three different scenarios are considered at the simulation level in order to compare the displacement characteristics of square shaped diaphragms, (a) a diaphragm with no corrugations (a flat diaphragm), which is clamped only from two sides, (b) a flat diaphragm, which is clamped from all the sides, and (c) a corrugated diaphragm, which is clamped from all the sides. The main difference in these three designs is the use of different mechanical boundary conditions for each design, while keeping the electrical boundary conditions and the material types unchanged. 


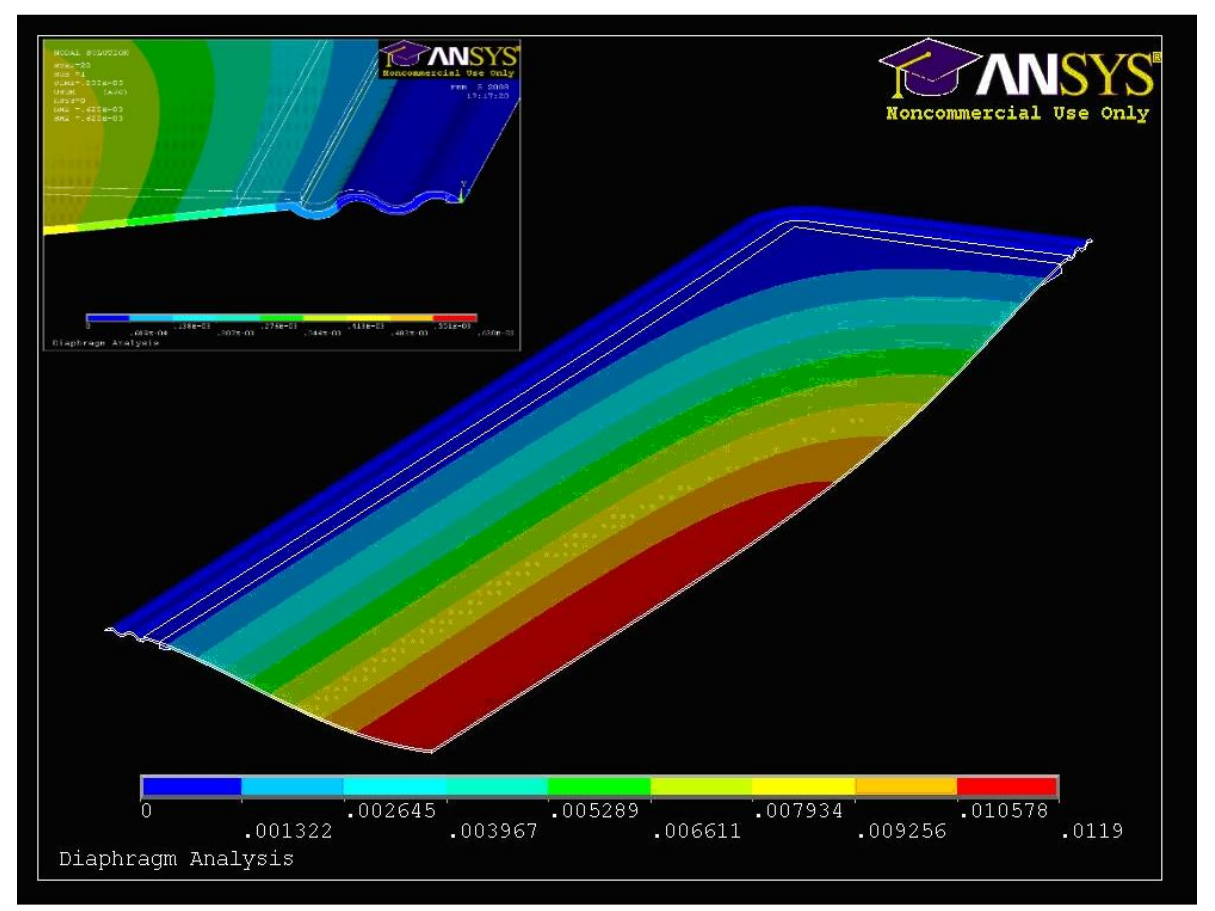

Figure 5. Finite Element Analysis (FEA) of electrostatically actuated, corrugated diaphragm design using ANSYS tools. Quarter-symmetry is exploited to reduce the simulation time and CPU usage. Two wavelengths $(2 \times l)$ of corrugations are used for initial analysis. Dimensions of the quarter-diaphragm segment considered are $1000 \mu \mathrm{m} \times 2 \mu \mathrm{m} \times 250 \mu \mathrm{m}(\mathrm{L} / 2$ $\times \mathrm{H} \times \mathrm{W} / 2)$.

As it can be seen from Figure 5, the symmetry condition is exploited in order to reduce the simulation time, while achieving a complete set of results. For all three scenarios above, quarter-symmetry is used, which means only a quarter of the design is simulated, since the square shaped diaphragm has two axes of symmetry.

The displacements achieved by a mid-node of the diaphragm, for each scenario, is plotted and compared in Figure 6. As expected, quadratic shaped curves are observed since the relationship between the applied voltage and the displacement of the diaphragm is quadratic in nature, when the diaphragm is actuated electrostatically. ${ }^{3,11}$

Comparatively higher displacements are achieved when the diaphragm is clamped only in two sides. This is because the stress levels are low in the middle section of the diaphragm as it is free to move from sides. However, this sort of a diaphragm is not appropriate for a micropump as it can create some leaking through the sides. When the diaphragms are fully clamped, corrugated diaphragm structure provides more displacement compared to a flat diaphragm. By fine tuning the design parameters for sinusoidal corrugations, even more displacement can be possibly achieved.

\section{CONCLUSION}

The design and analysis of microdiaphragms for microfluidic devices is presented in this paper. The importance in designing diaphragms, that require less power to operate especially in nano-scale drug delivery and similar applications is highlighted. The effect of incorporating corrugated profiles into diaphragms is illustrated and different scenarios are analysed to highlight that corrugated diaphragms achieve higher displacement over flat diaphragms for the same electrostatic field.

Consequently, some important future research is identified in order to improve the diaphragm performance. Design the diaphragm to be more flexible by carefully choosing materials that have mechanical properties, that are best suited for higher flexibility, while maintaining the structural stability, is one important step in this process. 


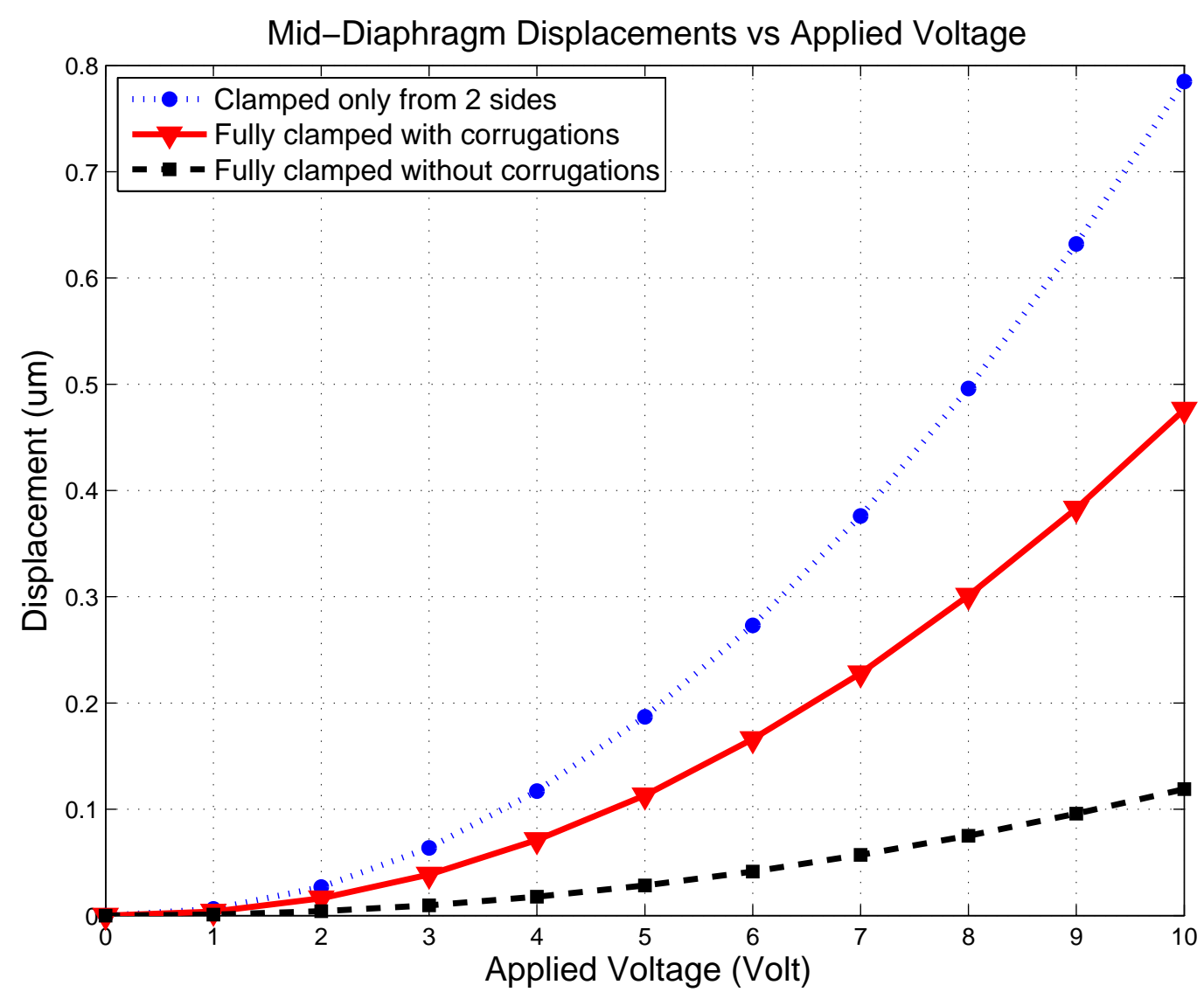

Figure 6. Comparison of displacements achieved by a mid-node of the diaphragm for the 3 different scenarios mentioned. Full dimensions of the diaphragms used are $2000 \mu \mathrm{m} \times 2 \mu \mathrm{m} \times 500 \mu \mathrm{m}(\mathrm{L} \times \mathrm{H} \times \mathrm{W})$.

Moreover, to achieve the best possible actuation, the addition of corrugated profiles needs to be analysed more carefully and in depth, concentrating more on the variations that can be introduced by the corrugation design parameters.

\section{ACKNOWLEDGMENTS}

The authors would like to thank the Australian Research Council (ARC) and the School of Electrical and Electronic Engineering, The University of Adelaide, Australia, for providing the necessary funding, facilities and the support to carry out this research.

\section{REFERENCES}

[1] D. W. Dissanayake, S. Al-Sarawi and D. Abbott, "Surface Acoustic Wave Device Based Wireless Passive Microvalve for Microfluidic Applications," Proc. of SPIE-BioMEMS and Nanotechnology III 6799, pp. 67990H: 1-10, December 2007.

[2] V. K. Varadan and V. V. Varadan, "Microsensors, micromechanical systems (MEMS), and electronics for smart structures and systems," Smart Materials and Structures 9, pp. 953-972, 2000.

[3] D. W. Dissanayake, S. Al-Sarawi and D. Abbott, "Surface Acoustic Wave Device Based Electrostatic Actuator for Microfluidic Applications," 2nd International Conference on Sensing Technology, pp. 381-386, November 2007.

[4] D. W. Dissanayake, A. C. Tikka, S. Al-Sarawi and D. Abbott, "Radio frequency controlled microvalve for biomedical applications," Proc. of SPIE-Smart Materials IV 6413, pp. 64130D: 1-13, 2007. 
[5] I. Jones, L. Ricciardi, L. Hall, H. Hansen, V. Varadan, C. Bertram, S. Maddocks, S. Enderling, D. Saint, S. Al-Sarawi and D. Abbott, "Wireless RF communication in biomedical applications," Smart Materials and Structures 17, pp. 015050: 1-10, 2008.

[6] C. S. Rangan, G. R. Sarma and V. S. V. Mani, Instrumentation Devices and Systems, Tata McGraw-Hill Pub. Comp. Ltd., New Delhi, 1983.

[7] N. Soin and B. Y. Majlis, "Development of perfect silicon corrugated diaphragm using anisotropic etching," Microelectronic Engineering 83, pp. 1438-1441, 2006.

[8] W. J. Wang, R. M. Lin, Y. Ren and X. X. Li, "Performance of a novel non-planer diaphragm for highsensitivity structures," Microelectronics Journal 34(9), pp. 791-796, 2003.

[9] M. D. Giovanni, Flat and Corrugated Diaphragm Design Handbook, Marcel Dekker Inc., New York, 2 ed., 1982.

[10] ANSYS Incorporation, "Home page." visited on 21/11/2007.

[11] M. N. Horenstein, J. A. Perreault and T. G. Bifano, "Differential capacitive position sensor for planar MEMS structures with vertical motion," Sensors and Actuators 80, pp. 53-61, 2000. 